\title{
ALGORITHMS FOR JOINT ESTIMATION OF ATTENUATION AND EMISSION IMAGES IN PET
}

\author{
Hakan Erdoğan \\ IBM TJ Watson Research Labs \\ PO Box 218 \\ Yorktown Heights, NY 10598
}

\author{
Jeffrey A. Fessler \\ 4415 EECS Bldg. \\ University of Michigan \\ Ann Arbor, MI 48109-2122
}

\begin{abstract}
In positron emission tomography ( $\mathrm{PET}$ ), positron emission from radiolabeled compounds yields two high energy photons emitted in opposing directions. However, often the photons are not detected due to attenuation within the patient. This attenuation is nonuniform and must be corrected to obtain quantitatively accurate emission images. To measure attenuation effects, one typically acquires a PET transmission scan before or after the injection of radiotracer. In commerically available PET scanners, image reconstruction is performed sequentially in two steps regardless of the reconstruction method: 1 . Attenuation correction factor computation (ACF) from transmission scans, 2. Emission image reconstruction using the computed ACFs. This two-step reconstruction scheme does not use all the information in the transmission and emission scans. Postinjection transmission scans contain emission contamination that includes information about emission parameters. Similarly, emission scans contain information about the attenuating medium. To use all the available information, we propose a joint estimation approach that estimates the attenuation map and the emission image simultaneously from these two scans. The penalized-likelihood objective function is nonconvex for this problem. We propose an algorithm based on paraboloidal surrogates that alternates between updating emission and attenuation parameters and is guaranteed to monotonically decrease the objective function.
\end{abstract}

\section{INTRODUCTION}

For a quantitatively accurate PET image, two scans are required: transmission and emission scans. Transmission scans are performed to estimate the attenuation characteristics of the medium. The attenuation information gathered from transmission scans are used to correct for its effects on the emission data to reconstruct emission images. Conventional method consists of linear processing (smoothing) of transmission data to obtain attenuation correction factors (ACFs) and multiplying the smoothed emission data with these factors to correct for the effects of attenuation [1]. Statistical penalized-likelihood methods reconstruct the attenuation map image with a local smoothing penalty and reproject them to obtain ACFs [2]. These ACFs are then used in the penalized-likelihood reconstruction of the emission data by incorporating them in the emission data sta- tistical model [3]. Both of these methods employ a sequential approach. First, ACFs are obtained from transmission scans and then emission data is reconstructed using the ACFs.

In this paper, we propose a different approach to image reconstruction which attempts to utilize all the information in transmission and emission scans. Post-injection transmission scans are corrupted by emission counts, so there is information about the emission parameters in the transmission scan. On the other hand, in an emission scan, there is information about the attenuation properties of the medium, since the measurements are affected by the attenuation in the body. Thus, to make optimal use of the information in these two scans, one can derive a joint objective function based on both scans to jointly estimate attenuation and emission parameters [4]. This approach should yield better results than the standard sequential estimation strategy.

\section{THE MODEL}

Let $\lambda=\left[\lambda_{1}, \ldots, \lambda_{p}\right]$ denote the vector of unknown emission counts originating from image pixels, and $\mu=\left[\mu_{1}, \ldots, \mu_{p}\right]$ be the vector of linear attenuation coefficients (having units of inverse length). Let $y^{T}=\left[y_{1}^{T}, \ldots, y_{N}^{T}\right]$ denote the vector of post-injection transmission scan counts, and $y^{E}=$ $\left[y_{1}^{E}, \ldots, y_{N}^{E}\right]$ denote the vector of emission scan counts. We assume that the $y_{i}^{T}$ and $y_{i}^{E}$ are realizations of statistically independent random variables having Poisson distributions and with expectations $\bar{y}_{i}^{T}$ and $\bar{y}_{i}^{E}$ :

$$
\begin{gathered}
\bar{y}_{i}^{T}(\lambda, \mu)=b_{i} e^{-l_{i}(\mu)}+k_{i} p_{i}(\lambda) e^{-l_{i}(\mu)}+r_{i}^{T}, \\
\bar{y}_{i}^{E}(\lambda, \mu)=p_{i}(\lambda) e^{-l_{i}(\mu)}+r_{i}^{E},
\end{gathered}
$$

for $i=1 \ldots N$, and where

$$
p_{i}(\lambda)=\sum_{j} a_{i j} \lambda_{j} \text { and } l_{i}(\mu)=\sum_{j} g_{i j} \mu_{j}
$$

where $a_{i j}$ represent the emission projection geometry including the detector efficiencies, and $g_{i j}$ represent the tomographic system geometry for attenuation. Here, $b_{i}$ are the time adjusted blank scan counts, $p_{i}$ and $l_{i}$ are the raw projections of true emission and attenuation parameters, $r_{i}^{T}$ and $r_{i}^{E}$ are background counts in their respective scans, $k_{i}$ is the fraction of emission counts in the transmission scan for each ray $i$, and $\varepsilon_{i}$ 's are the detector efficiencies. 
Our final goal is to estimate $\lambda$ from the measurements. However, the unknown attenuation map $\mu$ (or the ACFs) has to be estimated to get an accurate estimate of $\lambda$. The goal of any reconstruction algorithm is to improve the quality of the reconstructed emission image.

\section{SEQUENTIAL METHODS}

Conventional PET image reconstruction with post-injection transmission scans consists of the following steps $[5,6]$. First, scaled emission counts are subtracted from the transmission scan. Scaling accounts for deadtime, scan durations, radioactive decay and rod windowing to estimate the emission contamination accurately. An attenuation map $\hat{\mu}$ is reconstructed from the subtracted data next. Finally, attenuation correction factors are formed and applied to emission sinogram to reconstruct the emission image $\hat{\lambda}$.

Although this subtraction based approach might give satisfactory results for the brain scans where attenuation is almost uniform, it is suboptimal for thorax scans due to nonuniform attenuation. This method disregards measurement noise statistics, namely the Poisson nature of the measurement data. Subtraction further destroys Poisson statistics of transmission sinogram. This approach harms the reconstruction most for high attenuation rays, since the transmission counts are typically lower for those rays. Subtraction results in negatives in transmission sinogram which is problematic as well. Transmission scan data can be smoothed to reduce noise at the expense of reduced spatial resolution and artifacts in the emission image. Because of the noise problems, this method might require unreasonably long transmission scans for whole-body studies.

\section{JOINT ESTIMATION}

Joint estimation is theoretically more advantageous as compared to sequential methods since all the data is used to estimate all the unknown parameters [7]. In this method, we minimize one joint objective function to find the optimum values for $\mu$ and $\lambda$. We simply concatenate the measurements $y^{E}$ and $y^{T}$ to form the measurement vector and also $\lambda$ and $\mu$ to form the parameter vector. Since, emission and transmission counts are independent from each other, a joint penalized likelihood objective function can be written by summing up individual log-likelihoods and the individual penalty terms.

$$
\begin{gathered}
{\left[\begin{array}{c}
\hat{\lambda} \\
\hat{\mu}
\end{array}\right]=\underset{\lambda, \mu}{\arg \min } \Phi\left(\left[\begin{array}{l}
\lambda \\
\mu
\end{array}\right] ;\left[\begin{array}{l}
y^{E} \\
y^{T}
\end{array}\right]\right),} \\
\Phi\left(\left[\begin{array}{c}
\lambda \\
\mu
\end{array}\right] ;\left[\begin{array}{l}
y^{E} \\
y^{T}
\end{array}\right]\right)=\Phi^{T}(\mu, \lambda)+\Phi^{E}(\mu, \lambda),
\end{gathered}
$$

where $\Phi^{T}(\mu, \lambda)$ and $\Phi^{E}(\mu, \lambda)$ are penalized-likelihood objective functions for transmission and emission scans respectively:

$$
\Phi^{T}(\mu, \lambda)=\sum_{i=1}^{N} h_{i}^{T}\left(l_{i}(\mu), p_{i}(\lambda)\right)+\beta_{\mu} R_{\mu}(\mu)
$$

and

$$
\Phi^{E}(\mu, \lambda)=\sum_{i=1}^{N} h_{i}^{E}\left(l_{i}(\mu), p_{i}(\lambda)\right)+\beta_{\lambda} R_{\lambda}(\lambda)
$$

where we view the marginal negative log-likelihood functions $h_{i}^{T}$ and $h_{i}^{E}$ as a function of the projections $l_{i}$ and $p_{i}$. The objective function only depends on the parameters $\lambda$ and $\mu$ through their projections $p_{i}$ and $l_{i}$ :

$$
h_{i}^{T}\left(l_{i}, p_{i}\right)=\bar{y}_{i}^{T}\left(l_{i}, p_{i}\right)-y_{i}^{T} \log \bar{y}_{i}^{T}\left(l_{i}, p_{i}\right)
$$

and

$$
h_{i}^{E}\left(l_{i}, p_{i}\right)=\bar{y}_{i}^{E}\left(l_{i}, p_{i}\right)-y_{i}^{E} \log \bar{y}_{i}^{E}\left(l_{i}, p_{i}\right) .
$$

Note that the mean values of two measurements $\bar{y}_{i}^{E}$ and $\bar{y}_{i}^{T}$ both contain the emission and attenuation projections $l_{i}$ and $p_{i}$ in them. In general the objective is nonconvex and the global minimization is hard.

\section{OPTIMIZATION METHOD}

We propose to achieve a local minimum of the objective function $\Phi$ by alternatingly updating the emission and attenuation images. We make use of the paraboloidal surrogates [8] idea to obtain an algorithm that monotonically decreases the objective function assuring convergence to a local minimum. First we make this observation: Once either $\lambda$ or $\mu$ is fixed, the form of the functions $h_{i}^{T}$ and $h_{i}^{E}$ are similar to their counterparts in penalized-likelihood estimation for the other parameter. We use this observation to derive the following algorithm.

We describe the algorithm using induction. Initial attenuation and emission images $\mu^{0}$ and $\lambda^{0}$ are found using the conventional sequential method. Say, $\mu=\mu^{n}$ and $\lambda=\lambda^{n}$ are the current estimates of two parameters obtained after iteration $n$. We fix the terms $\lambda^{n}$ at their current value and allow only the terms $\mu$ to change. Our aim is to find:

$$
\mu^{n+1}=\underset{\mu \geq 0}{\arg \min } \Phi^{E}\left(\mu, \lambda^{n}\right)+\Phi^{T}\left(\mu, \lambda^{n}\right) .
$$

We denote the current values of the projections as $p_{i}^{n} \triangleq$ $p_{i}\left(\lambda^{n}\right)$ and $l_{i}^{n} \triangleq l_{i}\left(\mu^{n}\right)$. The form of the mean values for both scans when the $\lambda$ terms are fixed and assumed constant is:

$$
\bar{y}_{i}^{S}=A_{i}^{S} e^{-l_{i}}+B_{i}^{S}, \text { for } S \in\{T, E\} .
$$

where $A_{i}^{S}=A_{i}^{S}\left(p_{i}^{n}\right)$ and $B_{i}^{S}$ are constants independent of $l_{i}$. Furthermore $A_{i}^{S}>0$ and $B_{i}^{S} \geq 0$ for both scans. These conditions satisfy the conditions in Theorem 1 of [8], and we can find surrogate parabolas $q_{i}^{T}\left(l_{i}\right)$ and $q_{i}^{E}\left(l_{i}\right)$ that lie above $h_{i}^{T}\left(l_{i}\right)$ and $h_{i}^{E}\left(l_{i}\right)$ and tangent to them at the current projection $l_{i}^{n}$. The sum of these two parabolas $q_{i}\left(l_{i}\right) \triangleq$ $q_{i}^{T}\left(l_{i}\right)+q_{i}^{E}\left(l_{i}\right)$ is also a parabola. Once the curvature and gradient of the parabola is determined, they can be fed into the paraboloidal surrogates coordinate descent (PSCD) [8] or paraboloidal surrogates ordered subsets (OSTR) [9] algorithms to update the attenuation parameters to obtain the next iterate $\mu^{n+1}$. 
Similarly, we next fix the attenuation map values $\mu^{n+1}$ and allow only the $\lambda$ parameters to change to minimize the objective function:

$$
\lambda^{n+1}=\underset{\lambda \geq 0}{\arg \min } \Phi^{E}\left(\mu^{n+1}, \lambda\right)+\Phi^{T}\left(\mu^{n+1}, \lambda\right) .
$$

When the attenuation parameters are fixed, the form of the means for both scans is as follows:

$$
\bar{y}_{i}^{S}=C_{i}^{S} p_{i}+D_{i}^{S}, \text { for } S \in\{T, E\} .
$$

Here once again $C_{i}^{S}=C_{i}^{S}\left(l_{i}^{n+1}\right)$ and $D_{i}^{S}$ are constants independent of $p_{i}$. The objective function viewed as only a function of $\lambda$ (or $p_{i}$ 's) is convex, and strictly convex if $y_{i}^{S}>0$. Hence, the form of (2) makes it possible for $h_{i}^{E}\left(p_{i}\right)$ and $h_{i}^{T}\left(p_{i}\right)$ (viewed as functions of $p_{i}$ only) to satisfy the conditions of Theorem 1 in [8]. Hence, similar to the attenuation parameter update, one can obtain parabolas that lie above these $h$ functions and tangent to them at the current iterate $p_{i}^{n}[10]$. After the parabolas are obtained, it is easy to implement a PSCD algorithm similar to [10].

This joint estimation algorithm is easy to implement and results in a very fast algorithm. Once the gradient and curvatures of the parabolas are determined, the problem turns into a penalized weighted least squares type optimization problem and the computations of updates become very fast $[8,10]$.

\section{THE CURVATURES}

The curvatures of the surrogate parabolas $q($.$) play an im-$ portant role in the paraboloidal surrogates algorithm. The curvature is the only free parameter that has to be chosen to ensure monotonicity. We have shown [8] that the optimum curvature that results in the widest parabola yet ensuring monotonicity in the transmission scan can be given by (dropping sub and super scripts):

$$
c= \begin{cases}{\left[2 \frac{h(0)-h\left(l^{n}\right)+l^{n} \dot{h}\left(l^{n}\right)}{\left(l^{n}\right)^{2}}\right]_{+},} & l^{n}>0, \\ {[\ddot{h}(0)]_{+},} & l^{n}=0 .\end{cases}
$$

It can be shown that for the attenuation parameter update, when the mean is given by (1), we get the following optimum curvature expression $c^{\text {opt }}(l, A, B, y)=$

$$
\begin{cases}{\left[\frac{2}{l^{2}}\left(A f(l)-y \log \frac{A+B}{A e^{-l}+B}+\frac{y l A e^{-l}}{A e^{-l}+B}\right)\right]_{+},} & l>0, \\ {\left[A\left(1-\frac{B y}{(A+B)^{2}}\right)\right]_{+},} & l=0,\end{cases}
$$

where $f(l)=1-e^{-l}-l e^{-l}$. So, the optimum curvature for updating $\mu$ becomes: $c_{\text {joint }}^{\text {opt }}(l)=c^{\text {opt }}\left(l_{i}^{n}, A_{i}^{T}, B_{i}^{T}, y_{i}^{T}\right)+$ $c^{\mathrm{opt}}\left(l_{i}^{n}, A_{i}^{E}, B_{i}^{E}, y_{i}^{E}\right)$ where:

$$
\begin{gathered}
A_{i}^{T}=b_{i}+k_{i} p_{i}^{n}, \quad A_{i}^{E}=p_{i}^{n}, \\
B_{i}^{T}=r_{i}^{T}, \quad B_{i}^{E}=r_{i}^{E} .
\end{gathered}
$$

For the emission parameter $\lambda$, the marginal likelihood function $h_{i}^{E}\left(p_{i}\right)$ is such that when $r_{i}^{E} \rightarrow 0, h_{i}^{E}(0) \rightarrow \infty$ and $\dot{h}_{i}^{E}(0) \rightarrow-\infty$. Even when $r_{i}^{E}$ is greater than zero but small (typically it is around $5-10 \%$ of the mean), $h_{i}^{E}(0)$ can be very large. Since the optimum curvature uses the value at zero as the touching point for the surrogate and the original function, it results in unnecessarily narrow surrogates in the emission case. It is advantageous to limit the feasible region for $\lambda_{j}$ 's (and hence $p_{i}$ 's) at each iteration to $\left[\gamma^{n} \lambda_{j}^{n}, \infty\right)$ where $0 \leq \gamma^{n}<1$ is a parameter that controls the shrinkage of the feasible region. Choosing $\gamma^{n}>0$ enables wider curvature surrogates which will result in faster convergence. However, as $n$ increases, it is desirable to decrease $\gamma^{n}$ to zero, so that the correct feasible region $[0, \infty)$ is achieved in the end. When the feasible region is limited, we get the following optimum curvature:

$c= \begin{cases}{\left[2 \frac{h\left(\gamma^{n} p^{n}\right)-h\left(p^{n}\right)+p^{n} \dot{h}\left(p^{n}\right)\left(1-\gamma^{n}\right)}{\left(p^{n}\right)^{2}\left(1-\gamma^{n}\right)^{2}}\right]_{+},} & p^{n}>0, \\ {[\ddot{h}(0)]_{+},} & p^{n}=0 .\end{cases}$

After some calculations, when the mean is given by (2), we get the following curvature $c^{\text {opt }}(p, C, D, y)=$

$$
\begin{cases}\frac{2 y}{\left(1-\gamma^{n}\right)^{2} p^{2}}\left(\log \frac{C p+D}{C \gamma^{n} p+D}-\frac{\left(1-\gamma^{n}\right) C p}{C p+D}\right), & p>0 \\ y C^{2} / D^{2}, & p=0 .\end{cases}
$$

Then, the optimum curvature for the joint estimation is as follows: $c_{\text {joint }}^{\text {opt }}\left(p_{i}^{n}\right)=c^{\text {opt }}\left(p_{i}^{n}, C_{i}^{T}, D_{i}^{T}, y_{i}^{T}\right)+$ $c^{\mathrm{opt}}\left(p_{i}^{n}, C_{i}^{E}, D_{i}^{E}, y_{i}^{E}\right)$ where:

$$
\begin{gathered}
C_{i}^{T}=k_{i} e^{-l_{i}^{n+1}}, \quad C_{i}^{E}=e^{-l_{i}^{n+1}}, \\
D_{i}^{T}=b_{i} e^{-l_{i}^{n+1}}+r_{i}^{T}, \quad D_{i}^{E}=r_{i}^{E} .
\end{gathered}
$$

Once the curvatures are determined, the surrogate paraboloids are determined and the optimization can be performed on the surrogate paraboloidal function.

\section{CONCLUSION}

We propose a new joint estimation algorithm for estimating attenuation and emission images from transmission and emission scans. The method is based on minimizing a joint objective function that contains terms from both scans with respect to attenuation and emission parameters. We use an alternating optimization scheme where we minimize one set of parameters at a time fixing the values of the other set. This results in a fast and efficient algorithm that guarantees monotonicity. The joint estimation approach is theoretically more accurate and uses all the available information to estimate all the parameters at once unlike current sequential approaches.

There might be other ways to minimize the objective function such as sequentially updating $\left(\lambda_{1}, \mu_{1}\right),\left(\lambda_{2}, \mu_{2}\right)$, $\ldots,\left(\lambda_{p}, \mu_{p}\right)$. This method might converge faster, but it is harder to implement and per iteration costs are higher. Our alternating optimization approach is faster, simpler and eas. ier to implement.

There are some challenges in using this method for PET image reconstructions. If random coincidences are pre-subtracted, the measurements are no longer Poisson, so other 
models such as Shifted Poisson [11] should be used. Since the emission distribution inside the body changes with time due to metabolism, methods to better estimate the emission contamination should be found. Obtaining good iniial estimates is also important since the joint problem is not globally convex and there might be multiple minima. The choice of the penalty hyperparameters $\beta$ 's affects the reconstructions considerably and their effect is not understood as well as the single image reconstruction case where there are approximations to estimate the spatial resolution properties of the reconstructed images[12]. In the sequential methods, the resolution mismatch between ACFs and emission data causes artifacts in the emission images $[1,13]$. In the joint estimation method, this problem affects the emission images as well. Finally, although theoretically joint estimation seems more attractive and enables use of all the information in PET scans, it remains to demonstrate that it outperforms a good sequential approach based on approximate statistical methods.

\section{REFERENCES}

[1] A. Chatziioannou and M. Dahlbom, "Detailed investigation of transmission and emission data smoothing protocols and their effects on emission images," IEEE Tr. Nuc. Sci., vol. 43, no. 1, pp. 290-4, February 1996.

[2] H. Erdoğan, Statistical image reconstruction algorithms using paraboloidal surrogates for PET transmission scans, PhD thesis, Univ. of Michigan, Ann Arbor, MI, 48109-2122, Ann Arbor, MI., July 1999.

[3] E. Ü. Mumcuoğlu, R. M. Leahy, and S. R. Cherry, "Bayesian reconstruction of PET images: methodology and performance analysis," Phys. Med. Biol., vol. 41, no. 9, pp. 1777-1807, September 1996.

[4] J. A. Fessler and E. P. Ficaro, "Maximum likelihood transmission image reconstruction for overlapping transmission beams," in Proc. IEEE Nuc. Sci. Symp. Med. Im. Conf., 1999.

[5] M. E. Daube-Witherspoon, R. E. Carson, and M. V. Green, "Post-injection transmission attenuation measurements for PET," IEEE Tr. Nuc. Sci., vol. 35, no. 1, pp. 757-761, February 1988.

[6] R. E. Carson, M. E. Daube-Witherspoon, and M. V. Green, "A method for postinjection PET transmission measurements with a rotating source," J. Nuc. Med., vol. 29 , no. 9, pp. 1558-1567, September 1988.

[7] H. Erdoğan and J. A. Fessler, "Joint estimation of attenuation and emission images from PET scans," in Proc. IEEE Nuc. Sci. Symp. Med. Im. Conf., 1999. To appear.

[8] H. Erdoğan and J. A. Fessler, "Monotonic algorithms for transmission tomography," IEEE Tr. Med. Im., vol. 18, no. 9, pp. 801-14, September 1999.

[9] H. Erdoğan and J. A. Fessler, "Ordered subsets algorithms for transmission tomography," Phys. Med. Biol., vol. 44, no. 11, pp. 2835-51, November 1999.
[10] J. A. Fessler and H. Erdoğan, "A paraboloidal surrogates algorithm for convergent penalized-likelihood emission image reconstruction," in Proc. IEEE Nuc. Sci. Symp. Med. Im. Conf., volume 2, pp. 1132-5, 1998.

[11] M. Yavuz and J. A. Fessler, "New statistical models for randoms-precorrected PET scans," in Information Processing in Medical Im., J. Duncan and G. Gindi, editors, volume 1230 of Lecture Notes in Computer Science, pp. 190-203, Springer Verlag, Berlin, 1997.

[12] J. A. Fessler and W. L. Rogers, "Spatial resolution properties of penalized-likelihood image reconstruction methods: Space-invariant tomographs," IEEE Tr. Im. Proc., vol. 5, no. 9, pp. 1346-58, September 1996.

[13] H. Erdoğan and J. A. Fessler, "Statistical image reconstruction methods for simultaneous emission/transmission PET scans," in Proc. IEEE Nuc. Sci. Symp. Med. Im. Conf., volume 3, pp. 1579-83, 1996. 